\title{
Propuesta de perfil del docente siglo xxi
}

\section{Teacher profile proposal xxi century}

DOI: $10.46932 / \mathrm{sfjdv2n5-047}$

Received in: Oct 1st, 2021

Accepted in: Dec 30th, 2021

\author{
Mg. Abraham Simeón Ojeda Torres \\ Maestría con mención en Docencia y Gestión Educativa. \\ Unidad de Gestión Educativa Local de Morropón - distrito Morropón, provincia Morropón, \\ departamento Piura. Mza. V Lote 8 A.H. Los Almendros, distrito Castilla, departamento Piura. \\ E-mail: aojedatorres@yahoo.es \\ MSc. Patricia Mercedes Tapia Macías \\ Abogada. Magíster en Ciencias Internacionales y Diplomacia. Guayaquil, Ecuador. \\ E-mail: patty_tapia56@yahoo.com \\ MSc. Sonia Carmina Venegas Paz \\ Comunicadora Social y Docente. MSc. en Ciencias Internacionales y Diplomacia. Trabajo en la \\ Universidad de Guayaquil. \\ E-mail: punto_maximo_77@hotmail.com
}

\begin{abstract}
RESUMEN
El objetivo de la presente propuesta es identificar las características esenciales que debe considerarse al momento de escoger o formar personas en docencia para el presente siglo XXI. Siendo sus objetivos específicos: comprender los modelos educativos, conceptuar nuevos escenarios de enseñanzaaprendizaje, identificar tecnologías emergentes en la era digital, proponer nuevos perfiles del modelo docente para el siglo XXI, promover el respeto por las diferencias culturales en el marco de la educación interculturalidad y promoción del desarrollo del pensamiento crítico, propositivo y constructivo. Para este diagnóstico, en relación a los escenarios educativos y la presencia de las nuevas tecnologías, se revisa el estado del arte en relación a la realidad educativa en general. Como resultado del análisis de la bibliografía estudiada, se tiene un acercamiento hacia una propuesta sobre el perfil de un docente del siglo XXI. Se propone seis ejes para definir al docente del siglo XXI. Por otro lado, es necesario poner en práctica el desarrollo del pensamiento crítico, constructivo y propositivo; el mismo que a partir de un acto de conciencia de su propia realidad, permitirá al estudiante, formular propuestas coherentes con la construcción de una nueva sociedad en la que su rol protagónico, se hace perceptible, desde los distintos estadios de su desarrollo.
\end{abstract}

Palabras clave: Docente, siglo XXI, educación.

\section{ABSTRACT}

The objective of this proposal is to identify the essential characteristics that must be considered when choosing or training people in teaching for the present 21 st century. Being its specific objectives: understanding educational models, conceptualizing new teaching-learning scenarios, identifying emerging technologies in the digital age, proposing new profiles of the teaching model for the $21 \mathrm{st}$ century, promoting respect for cultural differences in the framework of education interculturality and 
promotion of the development of critical, purposeful and constructive thinking. For this diagnosis, in relation to educational settings and the presence of new technologies, the state of the art is reviewed in relation to educational reality in general. As a result of the analysis of the bibliography studied, there is an approach towards a proposal on the profile of a teacher of the XXI century. Six axes are proposed to define the teacher of the XXI century. On the other hand, it is necessary to put into practice the development of critical, constructive and purposeful thinking; the same one that from an act of awareness of their own reality, will allow the student to formulate proposals consistent with the construction of a new society in which their leading role becomes perceptible, from the different stages of their development.

Keywords: Teacher, XXI century, education.

\section{INTRODUCCIÓN}

La UNESCO (2020) en su portal expresa que "los docentes representan una de las fuerzas más sólidas e influyentes con miras a garantizar la equidad, el acceso y la calidad de la educación". En los distintos foros de la última década se ha reiterado que, los docentes son la clave del desarrollo mundial sostenible. Si esto último es una aseveración, ¿por qué no se logra los cambios deseados? Aún es visible que "su formación, contratación, permanencia, estatus y condiciones de trabajo son temas que siguen siendo preocupantes" (consultado 13 de septiembre de 2020).

Para identificar las características del docente en el presente siglo, es importante indagar fuentes bibliográficas obtenidas desde los foros internacionales sobre educación, de Procedia - Social and Behavioral Sciencies (ScienceDirect), de ELSEVIER, Base de Datos de Scopus, y más bases, con el fin de fundamentar adecuadamente la propuesta. Los cambios son vertiginosos, lo que hace necesario considerar la pregunta rectora: ¿Cuáles son las características del docente en el presente siglo XXI frente a los cambios en los escenarios educativos inducidos por la presencia de la tecnología emergente y las tecnologías de información y comunicación? Teniendo como problemas específicos los siguientes: ¿Qué características deben tener los modelos educativos en el presente siglo? ¿Cuáles son las innovaciones que están produciendo las nuevas tecnologías en los escenarios de enseñanza-aprendizaje e investigación? ¿Cuáles podrían ser las características del perfil del docente del presente siglo XXI?

Por tal motivo, el objetivo de la presente propuesta es identificar las características esenciales que debe considerarse al momento de escoger o formar personas en docencia para el presente siglo XXI, considerando los modelos educativos pertinentes, nuevos escenarios educativos, las innovaciones tecnológicas y perfiles académicos exigidos en la globalización, dentro del marco de la interculturalidad y sus implicancias en relación consciente con cada una de las diferentes manifestaciones culturales.

Siendo sus objetivos específicos: comprender los modelos educativos, conceptuar nuevos escenarios de enseñanza- aprendizaje, identificar tecnologías emergentes en la era digital, proponer 
nuevos perfiles del modelo docente para el siglo XXI, promover el respeto por las diferencias culturales en el marco de la educación interculturalidad y promoción del desarrollo del pensamiento crítico, propositivo y constructivo.

Este trabajo de investigación tiene gran importancia, puesto que todavía desde la escuela hasta la educación superior, se cuentan con docentes que aprendieron con docentes del siglo XIX y estos a su vez, si llegaron a ser educadores los hicieron con esos rezagos de la praxis del conductismo y cognitivismo arraigados en los estamentos educativos, en su actualización buscaron inmigrar hacia un constructivismo naciente en Latinoamérica, hasta que en el siglo XX con la presencia de las tecnologías de información y comunicación intentaron enseñar bajo un paradigma instruccionista, es decir, una clase con tecnología, que es la generación que heredó el siglo XXI y que al desear practicar a ser docente tuvo que migrar hacia la tecnología que de lo básico aún le cuesta salir, siendo la tecnología una herramienta para casos transcendentales, mientras la pizarra de tiza ha sido reemplazada por la pizarra acrílica.

Por otro lado, es importante considerar el perfil del docente en cuanto a ser intercultural, que pretende constituirse en ejemplo de vida social, que rescata y valora su propia cultura o, en su defecto, la cultura del contexto en el que se desempeña, bajo el paradigma del respeto por las distintas manifestaciones, a partir del reconocimiento de su identidad cultural.

Así también, el desarrollo del pensamiento crítico, propositivo constructivo, como un proceso intelectual disciplinado y consciente que genera mayor creatividad en las acciones de respuesta ante los problemas que la vida ofrece al ser humano.

\section{DESARROLLO}

Para este diagnóstico, en relación a los escenarios educativos y la presencia de las nuevas tecnologías, se revisa el estado del arte en relación a la realidad educativa en general y en algunos casos se puntualizará en la educación superior como una culminación de procesos de calidad que deben cumplirse para que los estándares del perfil docente sean más comprendidos y asimilados por la sociedad en general. Asimismo, considera la importancia del uso de las tecnologías emergentes y las innovaciones en torno a los procesos de enseñanza aprendizaje, necesarios para reunir insumos para una propuesta acerca del docente siglo XXI.

Comencemos considerando aspectos de la historia de la educación, especialmente la educación superior, para comprender las raíces de los procesos académicos y la necesidad de cambios urgentes desde la modernidad y la globalización. Suayter, I., y Carrera, A. (2008) Universidad Nacional de Tucumán, Argentina, presentaron los modelos de la educación superior mediante una historiografía, ubicando los modelos de educación superior tal como los conocemos hoy desde el siglo XXI, donde destaca la 
concepción de la Edad Media, al referirse a Latinoamérica. Cita a Bruner, J. (1990) explicando que surge como "empresa educativa...surge por iniciativa de la oligarquía, el Estado y los consejos universitarios" (p. 6) y, cita a Pérez Lindo, A. (1999) como una "universidad profesionalista". Permitiendo deducir que este modelo inicial latinoamericano carecía de estrategias académicas y políticas, impidiendo el desarrollo del conocimiento con énfasis en lo práctico de la profesión, lográndose entre 1912 y 1929, una universidad más democrática y moderna.

Martínez Boom, A. y Orozco Tabares, J. (2015) Universidad de Salamanca, en contraposición a la investigación del párrafo anterior, expresando su percepción de la historia de la universidad norteamericana y sus modelos institucionales como "esquizofrenia institucional combinado con un descontrol controlado" (p. 154). Explican la cosmovisión y crecimiento durante el siglo XX, entre Europa Occidental y América del Norte, caracterizándose por políticas dedicadas a la divulgación del conocimiento con enfoque científico, tecnológico y calidad educativa que fomentan articulaciones interinstitucionales con criterios de pertinencia, calidad e internacionalización (Martínez y Orozco, 2015, p. 163); su enfoque sistemático les permite concluir con una descripción de la “...reconfiguración de la universidad con gestión, aprendizajes, tercerización y empresarización de su institucionalidad” (p. 167).

En Ecuador, Flores-Montiel, M.I.; Novillo, C.; Venegas-Paz, S.; Cadena-García, D.; NoroñaMedina, J. (2017) Universidad de Guayaquil, Ecuador, abordan el problema de la articulación con los actores estatales, empresa privada e industria y centros de I+D+i, que afecta su institucionalidad. Mediante una metodología de investigación científica de caso (Robert Yin, 2003) con enfoque cualitativo y metodologías: bibliográfica documental, analítico-sintético y comparativo propone un modelo teórico contextualizado para la investigación y vinculación con la sociedad con responsabilidad social.

Bournissen Juan M. (2017) parte su investigación desde un modelo pedagógico aplicable a la plataforma virtual existente de la Universidad de la Plata. Luego del diagnóstico, centra su trabajo en el estudiante y aplica principios del conectivismo (Siemens, 2004); como opción válida de aplicación académica y su modelo pedagógico guía se deriva del modelo educativo institucional, de sus normas y procedimientos, metodologías áulicas, uso de tecnología, y la gestión docente. Para construir su propuesta, utiliza las fases del "modelo de diseño instruccional ADDIE (Análisis, Diseño, Desarrollo, Implementación y Evaluación) [...] Optando por una investigación de diseño y desarrollo de caso único" (Bournissen, 2017, p. 24). El resultado fue considerar una escuela (Facultad) como prototipo para la implementación.

Mendoza Gamiño A. (2017) Universidad de Alicante, en su tesis pone interés en la necesidad de compartir los saberes haciendo uso de tecnologías en la era digital mediante un "análisis de la teoría del aprendizaje, tecnologías nuevas y los medios expresivos en el contexto de la globalización” (p. 31). Es 
una investigación de enfoque teórico y desarrolla un modelo aplicativo en el ámbito pedagógico diseñando “[...] un modelo didáctico experimental” (Mendoza, 2017, p. 406).

Sánchez Prieto, J. C. (2018) plantea el "diseño de un modelo de adopción tecnológica para evaluar la aceptación de tecnologías móviles en el profesorado de primaria" (p. 1) y aborda problemas de la “adaptación de los contenidos a las necesidades individualizadas de los discentes, y la incapacidad para integrar recursos multimedia en los actores educativos" (p. 18). Su metodología es sistemática y se basa en el "modelo de aceptación de Davis", aplicando estudios pre-piloto con una población de docentes en formación universitaria y dirigida a niños de primaria, dando como resultado un modelo compuesto por nueve constructos, citados en las (pp. 52, 97, 107). Bournissen y Sánchez coinciden en la aplicación del modelo en una institución y luego generalizarla de acuerdo a los contextos institucionales, resaltando la integración institucional y un quehacer educativo conjugado con la tecnología.

La Tesis doctoral en la Universidad Balears de Sosa Neira E., A. (2018) presenta el diseño de un modelo para incorporar lo que denomina "tecnologías emergentes (TE)” (p. 9) en los procesos áulicos y generando "estrategias didácticas" (p. 15), donde los docentes son agentes de cambio. Sosa, aplica el diseño (DBR) que muestra una estrecha relación entre teoría y práctica generando nuevas expectativas en los aprendizajes y procesos de enseñanza en los entornos complejos actuales; propone, una guía práctica que sea aplicable en los colegios de Bogotá, Colombia, para aplicar TEs en las gestiones áulicas.

En la misma línea de trabajo de investigación, tenemos el modelo de desarrollo del aprendizaje a través del aprendizaje virtual, realizado por Pattanasith \& Rampai (2015). El grupo meta se relaciona con los estudiantes graduados de postgrados para proveer capacitación continua, dividiendo en grupo de control y grupo experimental, teniendo como herramientas forma de análisis y síntesis de datos, cuestionario de satisfacción y medición de habilidades de aprendizaje autodirigido. Teniendo como resultado un prototipo con el cual se espera mejorar el aprendizaje colaborativo y desarrollar habilidades de aprendizaje autodirigido con el uso de entorno de aprendizaje en línea (pp. 60-64).

Un criterio similar con Sosa también lo tiene Santos-Batista \& Márques (2019). Estos autores consideran la gestión de proyectos a través de conferencias sobre sistemas y tecnologías de información para el ámbito social y de salud, en la cual, en esta transformación digital aplicada a la educación, los estudiantes son los protagonistas. Mismos que usan dispositivos móviles, han desarrollado más autonomía y mecanismos de comunicación que facilitan los reportes, creando una relación entre docente y estudiantes. Su modelo se basa en dos aspectos: los agentes y las tecnologías de comunicación. Siendo una conclusión muy importante: cambiar los métodos de enseñanza podría aportar más mejoras al incentivo y la participación de los estudiantes (pp. 123-130). 
Con la misma perspectiva de una nueva educación, Xu-Song \& Wang (2020) presentan la aplicación de la capacitación y educación referentes a seguridad inteligente, enmarcados en la educación tecnológica. Su propuesta se dirige hacia una optimización educativa, para proponer un modelo de gestión en red de capacitación en seguridad inteligente y con esto fomentar una educación a través de una red remota y orientada a objetos. Consideran los autores que no solo debe pensarse en una enseñanza virtual sino dentro de las mismas instalaciones crear ambientes de estudio de manera remota que facilite los aprendizajes, lo que permitiría una convergencia de las varias tecnologías de redes que pueden ser de beneficio a la educación (pp. 1-5).

Halleemah Mafraq y Yasser Kotb (2019) the Saudi Arabia's, about "Higher Education Institutions, propose the Massive Online Open Courses (MOOCs) for this level of study" (p. 77). Esta publicación en la WebScience por la Association for Computing Machinery, cree en la importancia del aprendizaje donde docentes, estudiantes y academia pueden interrelacionarse y desarrollar el conocimiento a través de círculos de apoyo académico, obteniéndose efecto positivo en la autogestión y en el comportamiento de los participantes (Mafraq \& Kotb, 2019, pp. 77-82). Ahora bien, la conciencia colectiva percibe que las instituciones del estado, en especial Latinoamérica, son de bajo nivel académico, con limitado presupuesto, sin gestión de autonomía bien definida, bajo recurso para capacitación continua, investigación y desarrollo tecnológico sostenida y sustentable, siendo aplicable, motivante e imperioso considerar los MOOCs en plataformas gratuitas entre instituciones educativas.

Mejorar la educación en instituciones tecnológicas, los bachilleratos técnicos y los politécnicos, también son un reto, debe existir un diseño adecuado a la enseñanza de tecnología, cuánto más ahora que los jóvenes buscan carreras cortas y productivas (Voûte, Stappers, Giaccardi, Mooij, \& Boeijen, 2020). El fortalecimiento de una educación basada en proyectos (ABP) es una experiencia que los autores han considerado válida para mejorar la educación en ambientes de aprendizaje colaborativos. Estas innovaciones con ambiente tecnológico, pero con la práctica constante de proyectos, permite un mejor diseño curricular por competencias, al nivel de la exigencia del sistema, resolviendo problemas más complejos con la participación multidisciplinaria, o sea, resolver problemas de su contexto, uniéndose a los actores educativos, de investigación y vinculación (pp. 51-66).

Cátia Alves Goram Putnik, investigadora de la Universidad de Minho, manifiesta en su artículo "Experiential learning of CAD Systems interoperatility in social network-based education" (Goram Putnik, 2019), el objetivo del aprendizaje es guiar a los estudiantes a lo que ha denominado "zona de flujo... con los principios: proyecto-primero, justo a tiempo" (p. 209). Utilizando el modelo de educación basado en redes sociales y la plataforma Learning Factory para lograr un equilibrio entre los desafíos que se plantean durante la clase y sus habilidades que deben ser adquiridas como perfil de su carrera. Goram 
concluye que es importante crear desafíos y equilibrar las habilidades que deben ser adquiridas, a manera de escenarios y uso de internet provee roles donde la empresa con sus clientes y proveedores de servicio, se relacionan con la IES (clientes, estudiantes, investigadores) brindando soluciones o servicios de corretaje.

La metodología presentada por Goram, permitiría una enseñanza-aprendizaje activos, de construcción social, interconectados, con valores y centrado en los actores de la educación, comprometida e integrada a su contexto.

Hablar de la educación comprometida e integrada a su contexto, implicará también conocer el "enfoque intercultural" de la educación, plasmada en distintos currículos nacionales de educación, tal como se presenta en el Perú, teniendo como base la identidad cultural, que implica a su vez, tener en cuenta diversas consideraciones en torno al tema, así, Villegas (2017), en su artículo: "La importancia de rescatar la identidad cultural, ante un mundo globalizado", define la identidad cultural como el "conjunto de valores, tradiciones, símbolos, creencias y modos de comportamiento que funcionan como elementos dentro de un grupo social y que actúan para que los individuos que lo forman puedan fundamentar su sentimiento de pertenencia"

Uno de los pilares que sostiene la identidad cultural de un país o región es la relación que las personas mantienen con su patrimonio cultural local, pues éste posibilita la formación de ciudadanos conscientes de los valores de su patria chica y afianza el sentido de pertenencia hacia la comunidad en que se vive. En tal sentido la Educación Secundaria Básica es clave por su responsabilidad en el desarrollo de actitudes, conocimientos y valores en los adolescentes. (Gonzáles, 2004)

Al respecto, Molano (2007) en su libro "Identidad Cultural un concepto que evoluciona" señala: que el concepto de identidad cultural encierra un sentido de pertenencia a un grupo social con el cual se comparte rasgos culturales, como costumbres, valores y creencias. La identidad no es un concepto fijo, sino que se recrea individual y colectivamente y se alimenta de forma continua de la influencia exterior. En esta investigación pretende aportar a la discusión sobre desarrollo territorial con identidad cultural, a través de un breve recorrido sobre la evolución de los conceptos de cultura, identidad y patrimonio cultural, producto de debates internacionales que permiten establecer un lenguaje y prácticas universales consensuadas, para identificar un hecho cultural y darle una especificidad que lo hace único, distinto y lo convierte en un aporte al conjunto de la humanidad.

La UNESCO promueve el acceso y el ejercicio los derechos, la diversidad como centro de la acción pública en la garantía de los derechos, la ruptura de los estereotipos como una responsabilidad de política pública como factores esenciales del Desarrollo Sostenible, en nuestra meta de "No dejar a nadie atrás". 
Por su parte, Velarde (2014) afirma que el tema de las identidades culturales en el Perú es complejo y amplio, y estas discusiones resultarán importantes si la intención es hablar de desarrollo para todos. "Se necesitan muchos más esfuerzos desde el aparato gubernamental a nivel de políticas de reconocimiento que no sólo trabajen por la preservación de las lenguas y culturas de los pueblos originarios", sino que además se debe luchar por la "erradicación de los escenarios de discriminación centralistas". En este sentido, se deberá implementar "políticas tanto en el sector educativo como en la regulación de los medios de comunicación, en el uso de espacios públicos, en la operativización de las lenguas originarias y en la convivencia social en general"'.

En lo que respecta al desarrollo del pensamiento crítico - constructivo y propositivo, recordaremos a Paiget con su aporte: "El objetivo principal de la educación es crear personas capaces de hacer cosas nuevas, y no simplemente repetir lo que otras generaciones hicieron", o tal como lo dijera Hernández (2016): "es en la búsqueda de creación del conocimiento, donde nos ayuda la didáctica crítica, puesto que implica un aprendizaje activo y significativo, que potencia en el estudiante la reflexión, la curiosidad y el cuestionamiento.

Es meritorio reconocer el aporte de Scriven (2003) en relación al pensamiento crítico, entendido como el "proceso intelectualmente disciplinado de conceptualizar, aplicar, analizar, sintetizar y / o evaluar de manera activa y hábil la información recopilada o generada por la observación, la experiencia, la reflexión, el razonamiento o la comunicación, como guía para la creencia y la acción”.

Por último, en esta línea del estado del arte, se considera al equipo de Ena Voûte, en relación a "Innovating a Large Design Education Program at a University of Technology", mediante su enfoque cualitativo, con metodología sistemática y propositiva, hace historia, análisis y síntesis de la realidad institucional (p. 53). Propone un programa de educación dirigido a la universidad tecnológica, focalizada en la universidad tecnológica Delft, destacando un entrelazamiento entre la educación, la investigación, las prácticas en el contexto industrial y social más amplio (Voûte, Stappers, Giaccardi, Mooij, \& Boeijen, 2020, pp. 55, 56).

Ena Voûte et.al. por otro lado, plantea necesidades: recursos, infraestructura, nueva currícula ajustada al contexto, nuevas carreras y especialidades (p. 60) y concuerda con los autores anteriores, que en síntesis debe existir una sola IES articulada a la sociedad y a la industria, y agrega; los actores de la educación, docentes y estudiantes, deben ser parte de ese engranaje de la investigación, como valor agregado (pp. 64-66). Aumentar las redes y las relaciones en todos los ámbitos debe ser una prioridad para forjar nuevos escenarios académicos y ofertas más atractivas.

La fundamentación bibliográfica expuesta, nos permite hacer deducciones sobre la necesidad de modelos educativos acordes al mundo globalizado y exigente de la tecnología; identificar las tecnologías 
emergentes de la era digital que influyen en su quehacer institucional como centro educativo al servicio de la sociedad. Ahora, es necesario dirigir la atención hacia el perfil que requiere observarse en un docente que enfrenta estas nuevas innovaciones en el presente siglo XXI.

\section{RESULTADOS}

Como resultado del análisis de la bibliografía estudiada, se tiene un acercamiento hacia una propuesta sobre el perfil de un docente del siglo XXI.

Se propone seis ejes para definir al docente del siglo XXI:

1. Docentes que laboren en instituciones educativas con modelos educativos ancladas en la política pública, alineadas al marco de los Tratados y Convenios Internacionales.

2. Que tengan escenarios teórico - prácticos de enseñanza - aprendizaje con herramientas tecnológicas, acordes a las exigencias de la globalización.

3. Docentes que respondan de manera propositiva a los avances tecnológicos de innovación educativa con alto contenido de investigación, desarrollo e innovación (I+D+i).

4. Docentes conocedores e involucrados en los nuevos perfiles académicos de formación para el docente del Siglo XXI.

5. Perfil del docente con identidad cultural, comprometido e integrado al contexto de su desenvolvimiento y respetuoso de la interculturalidad.

6. Perfil del docente que promueve en sus estudiantes el desarrollo pensamiento crítico, constructivo y propositivo.

\section{CONCLUSIONES}

La presente investigación, es una mirada caleidoscópica más profunda y propone la construcción de una nueva visión de modelo educativo integral para las instituciones educativas, articulados y comprometidos con la industria, matriz productiva y el desarrollo económico del país (Cheong, 2016, p. 478), pero siempre ligado a un contexto cercano, el reconocimiento de sus características peculiares, el interafecto telúrico que se puede establecer en la relación hombre - medio, traducida en una identidad con su cultura y, el respeto por las demás manifestaciones, que le asignan el carácter de "intercultural".

Por otro lado, es necesario poner en práctica el desarrollo del pensamiento crítico, constructivo y propositivo; el mismo que a partir de un acto de conciencia de su propia realidad, permitirá al estudiante, formular propuestas coherentes con la construcción de una nueva sociedad en la que su rol protagónico, se hace perceptible, desde los distintos estadios de su desarrollo. 
La presente investigación considera que en relación a la era digital, es importante que se desarrollen modelos educativos interrelacionados e interinstitucionales, con fundamentación filosófica, epistemológica, ontológica, axiológica, pedagógica entre otras, con el fin de lograr sus objetivos institucionales, brindando un mayor alcance para la comunidad educativa y para que el quehacer del docente sea más acorde al siglo XXI en el cual vivimos (Tünnermann B., 2008).

Asimismo, las Tecnologías emergentes (Tes) deben ser consideradas y deben ser incorporadas a partir del modelo actual educativo que se tenga en los países latinoamericanos, que su gran mayoría está asentada todavía en el paradigma constructivista, antesala de la era digital. Esto es, formar redes internas e interinstitucionales fortalecidas, centrado en docencia, estudiante y contexto, incluyendo estudiantes graduados de las instituciones educativas. 


\section{REFERENCIAS}

Bournissen, J. (2017). Modelo pedagógico para la Facultad de estudios virtuales de la Universidad Adventista del Plata. Son Esponyol i Sa Garriga, Islas Baleares, España: Universidad Baliarica.

Cheong, H.-W. (2016). Education Model of Technology Management for Promoting SMEs to Hidden

$\begin{array}{llll}\text { Champion. } & \text { Procedia } & \text { Computer }\end{array}$ doi:https://doi.org/10.1016/j.procs.2016.07.123

Gonzáles Sáenz, O. J. (s.f.).

Gonzáles Sáenz, O. J. (2004). La formación de la identidad cultural. Una mirada desde una escuela. UNESCO-CUBA, 11.

Goram Putnik, C. (2019). Experiential learnig of CAD systems interoperability in social network based education. Memories of Congress 29th CIRP Design 2019 (CIRP Design 2019). Peer-review under responsability of the scientific committee of the CIRP Design Conference 2019(84), 209-214. doi:10.1016/j.procir.2019-07.002

Hernández Sandoval, M. C. (2016). Experiencia de Investigación en Acción, en el marco de la aplicación de una didáctica crítica. Santiago: Universidad del Bío-Bío. Escuela de Pedagogía en Historia y Geografía (Chile).

Mafraq, H., \& Kotb, Y. (2019). Maarefh - Proposed MOOCs' Platform for Saudi Arabia's Higher Education Institutions. ICIET 2019: Proceedings of the 2019 7th International Conference on Information and Education Technology, 19(03), 77-82. doi:https://doi.org/10.1145/3323771.3323828

Martínez Boom, A., \& Orozco Tabares, J. (2015). Aprendizaje y Empresa en la Universidad que viene. Historia y Educación. Ediciones de Salamanca(34), 153-168. doi:http://dx.doi.org/10.14201/hedu201534153168

Mendoza Gamiño, A. (2017). Los saberes de la Era Digital: aprendizaje, nuevas tecnologías y artes expresivas (estudio teórico y aplicación práctica). Alicante: Universidad de Alicante, Tesis doctoral.

Molano, O. L. (2007). Identidad cultural, un concepto que evoluciona. Bogotá-Colombia: Revista Ópera. Noroña-Medina, J. A., Cadena G., D., Lucero, C., \& Flores, M. I. (2017). Modelo de gestión de proyectos de grado para generar investigación científica Universitaria. Conocimiento para el desarrollo, 8(2).

Noroña-Medina, J. Á., FloresMontiel, M. I., Lucero Novillo, C., Venegas Paz, S., \& Cadena García, D. (enero-junio de 2017). Modelo de gestión de investigación científica articulando actores: estatales, privados, centros de investigación y academia. Conocimiento para el desarrollo, 8(1), 105-112.

Pattanasith, S., \& Rampai, N. (february de 2015). The Development Model of Learning though Virtual Learning Environments (VLEs) for Graduated Students, Department of Educational Technology, Faculty of Education, Kasetsart University. Procedia - Social and Behavioral Sciences, ELSEIVER-SCOPUS, 176(20), 60-64. doi:https://doi.org/10.1016/j.sbspro.2015.01.444

Sánchez Prieto, J. (2018). Diseño de un modelo de adopción tecnológica para evaluar la aceptación de tecnologías móviles en el profesorado de primaria. Salamanca, España: Universidad de Salamanca, Tesis doctoral.

Obtenido

de https://knowledgesociety.usal.es/sites/default/files/tesis/Tesis\%20Jose\%20Carlos\%20Sanchez\%20Prieto .pdf 
Santos, H., Batista, J., \& Márques, R. P. (2019). Digital transformation in higher education: the use of communication technologies by students. ELSEVIER - SCOPUS, 164, 123-130. doi:https://doi.org/10.1016/j.procs.2019.12.163

Scriven, M. y. (2003). Defining critical thinking. The Fundation for Critical Thinking.

Siemens, G. (2004). Conectivismo: una teoría de aprendizaje para la era digital. Semantics scholar, 10. Obtenido de https://pdfs.semanticscholar.org/05f1/adee187323d66beab226058b23a7416c3517.pdf

Sosa Neira, E. (2018). Diseño de un modelo de incorporación de tecnologáis emergentes en el aula (MITEA) paa la generación de estrategias didácticas por parte de los docentes. Islas Balear, España: Universitat de les Illes Balears, Tesis doctoral. Obtenido de https://dspace.uib.es/xmlui/bitstream/handle/11201/149058/Sosa\%20Neira_Edgar\%20Andres_COMPE NDI.pdf?sequence $=1 \&$ isAllowed $=\mathrm{y}$

Suayter, I., \& Carrera, A. (2008). Origen y evoluación de la Educación Superior. V Encuentro Nacional y II Latinoamericano La Universidad como objeto de investigación, 1-12.

Tünnermann B., C. (2008). Modelos educativos y académicos. Managua, Nicaragua: Editorial HISPAMER. Obtenido de https://educar.ec/jornada/docmodelo/Modelos\%20educativos\%20y\%20academicos.pdf

Velarde, J. (2014). La educación intercultural y el desarrollo humano en el Perú. Lima: IDEHPUCP.

Villegas, M. V. (3 de enero de 2017). https://www.milenio.com/opinion/varios-autores/universidadtecnologica-del-valle-del-mezquital/importancia-rescatar-identidad-cultural-mundo-globalizado.

Obtenido de La importancia de rescatar la identidad cultural, ante un mundo globalizado: https://www.milenio.com/opinion/varios-autores/universidad-tecnologica-del-valle-del-

mezquital/importancia-rescatar-identidad-cultural-mundo-globalizado

Voûte, E., Stappers, P., Giaccardi, E., Mooij, S., \& Boeijen, A. (2020). Innovating a Large Design Education Program at a University of Technology. She Ji: The Journal of Design, Economics, and Innovation. ScienceDirect-ELSEVIER-SCOPUS, 6(Issue 50-66. doi:https://doi.org/10.1016/j.sheji.2019.12.001

Xu, B., Song, S., \& Wang, D. (2020). Application of smart safety training and education in network teaching management. Safety Science, ScienceDirect, Elsevier-SCOPUS, 124, 104608. doi:https://doi.org/10.1016/j.ssci.2020.104608 